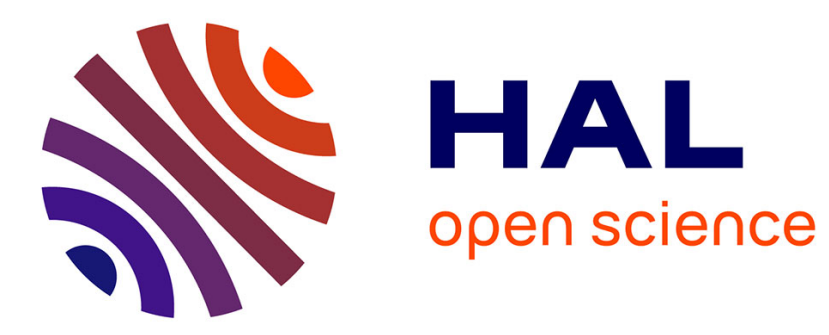

\title{
Human-Like Walking: Optimal Motion of a Bipedal Robot With Toe-Rotation Motion
}

Tlalolini Tlalolini David, Christine Chevallereau, Yannick Aoustin

\section{To cite this version:}

Tlalolini Tlalolini David, Christine Chevallereau, Yannick Aoustin. Human-Like Walking: Optimal Motion of a Bipedal Robot With Toe-Rotation Motion. IEEE/ASME Transactions on Mechatronics, 2011, 16 (2), pp.310-320. 10.1109/TMECH.2010.2042458 . hal-00483135

\section{HAL Id: hal-00483135 \\ https://hal.science/hal-00483135}

Submitted on 12 May 2010

HAL is a multi-disciplinary open access archive for the deposit and dissemination of scientific research documents, whether they are published or not. The documents may come from teaching and research institutions in France or abroad, or from public or private research centers.
L'archive ouverte pluridisciplinaire HAL, est destinée au dépôt et à la diffusion de documents scientifiques de niveau recherche, publiés ou non, émanant des établissements d'enseignement et de recherche français ou étrangers, des laboratoires publics ou privés. 


\begin{abstract}
Fast human walking includes a phase where the stance heel rises from the ground and the stance foot rotates about the stance toe. This phase where the biped becomes underactuated is not present during the walk of humanoid robots. The objective of this study is to determine if the introduction of this phase for a 3D biped robot is useful to reduce the energy consumed in the walking. In order to study the efficiency of this new gait, two cyclic gaits are presented. The first cyclic motion is composed of successive single support phases with flat stance foot on the ground, the stance foot does not rotate. The second cyclic motion is composed of single support phases that includes a sub-phase of rotation of the supporting foot about the toe. The single support phases are separated by a double support phase. For simplicity this double support phase is considered as instantaneous (passive impact). For these two gaits, optimal motions are designed by minimizing a functional torques cost. The given performances of actuators are taken into account. It is shown that for fast motion a foot rotation sub-phase is useful to reduce the functional cost. These gaits are illustrated with simulation results.
\end{abstract}

Index Terms-Biped robot, robot dynamics, Fully actuated robot, Newton-Euler algorithm, Cyclic walking gait, parametric optimization.

\section{INTRODUCTION}

The design and development of anthropomorphic robots with capability of walking naturally like human is one of the current greatest challenges of science. Research efforts has led in recent times to the development of remarkable anthropomorphic robots as the Honda Humanoid Robot introduced in 1997 [1] which could go up/down stairs. ASIMO appeared in 2000 [2], ${ }_{\text {h walks continuously while changing directions. }}$ JOHNNIE at the Technical University of Munich [3], which overall weight is about $40 \mathrm{~kg}$ and the height is $1.80 \mathrm{~m}$. HRP2 is another humanoid robot showing abilities to work with human [4]. Despite that each of these robots are noteworthy for their autonomy and interaction with their environment since these combine many desirable features needed to satisfy the dynamic bipedal locomotion close to human locomotion, they only execute flat-footed (fully-actuated phase) walking. From studies of human walking gait authors proved the fundamental role of the feet during the walking gait in double support phases and in single support phases. Thus, for human walking gait in single support, a rotation of the foot is observed with a partial contact of the sol with the ground, located between the heel and the toe, [5]. Furthermore it is shown that the feet, with joint torques at the ankle which are significant, play a role more important to insure an equilibrium of the biped than to help the locomotion, [6], [7] and [8]. Extending the analysis of walking with point feet, [9] has outlined a solution to the problem of walking with both fully-actuated and under-actuated phases for a planar biped robot with

David Tlalolini, Christine Chevallereau and Yannick Aoustin are with IRCCyN, UMR 6597 Centrale de Nantes, Université de Nantes, France e-mail : David.Tlal $\bar{\equiv}$ irccyn.ec-nantes.fr, Yannick.Aoustin@irccyn.ecnantes.fr, Christine.Chevallereau@irccyn.ec-nantes.fr non-trivial feet. In reference [10], solving an optimization problem considering under-actuated, fully-actuated and overactuated phases for planar motion, is shown that for fast motions the use of a foot rotation sub-phase (under-actuated phase) is significant to reduce the energy consumed during the walking. Then, it is extremely important and interesting to study the walking gait of an anthropomorphic biped with rotation feet. In this paper, the main obiective is to extend our analysis of optimal reference with foc $\overline{\overline{\bar{\nu}}}$ ation to optimal trajectories generation for an anthropomorphic robot to achieve an optimal fast motion. Therefore, the efforts are focused on the design of reference trajectories for a dynamically stable walking three-dimensional biped robot including foot rotation. In particular, in order to solve the under-actuation problem and to ensure the feasibility of the robot's motion during the foot rotation sub-phase, we chosen the geometric evolution of the robot [11], [12]. It corresponds to a motion compatible with the dynamic model so that the center of pressure $(C o P)$ is forced to remain strictly of the front limit of the stance foot, allowing the foot to rotate. The gait under study consists of successive single support phases separated by instantaneous double support phases. Single support phase is separated into two sub-phases, a flat foot sub-phase and a foot rotation sub-phase, in function of the biped velocity and the energy cost. Motions minimizing an integral criterion based on the vector of the square of the torques are defined for a gait. Furthermore, some constraints such as actuator performances and limits on the ground reaction force are taken into account. Section II presents the geometric description and dynamic model of the three-dimensional biped robot using NewtonEuler formulation. Section III is devoted to the development of the impact model for the instantaneous double support phase, adding Newton variables to define the velocity of the reference frame attached to the sole of the foot. The formulation of the optimization problem for optimal cyclic gait with and without foot rotation are defined in section IV. In the section V the various constraints and the cost function taking into account during the optimal processes are defined. The simulation results are presented. The conclu $\overline{\bar{\nu}}$, are given in section VI.

\section{Modeling OF THREE-DIMENSIONAL BIPED ROBOT}

\section{A. The biped}

Since a precise modeling of three-dimensional biped robot is particularly crucial for the development of dynamically stable trajectories to achieve an anthropomorphic motion, we considered an anthropomorphic biped robot which geometrical (dimensions of the bodies) and inertial (masses, positions of the centers of mass, moments and products of inertia of each body) distribution close to those of the human body. The humanoid construction is assumed to consist of seven rigid links connected by fourteen motorized joints to form a serial structure. This serial structure or open kinematic chain is 
further composed of two identical sub-chains called the legs, connected at the hip, and a body identified as a torso, which is not directly actuated as is depicted in figure 1. Each leg is composed of two massive links connected by a joint called knee. The link at the extremity of each leg is called foot which is connected at the leg by a joint called ankle. The general specifications of the biped robot in terms of size and DoF (degrees of freedom) are defined to imitate the human walk. Therefore, each ankle of the biped robot consists of the pitch and the yaw axes (flexion/extension and abduction/adduction) and one additional roll axis to take into account the foot twist rotation. The knees consist of the pitch axis (flexion/extension) and the hips consist of the roll, pitch and yaw axes (rotation, flexion/extension and abduction/adduction) to constitute a biped walking system of two 3-DoF ankles, two 1-DoF knees and two 3-DoF hips, table I. Each revolute joint is assumed to be independently actuated and ideal (frictionless). The action of the walking motion associates single support phases separated by impacts with full contact between the sole of the feet and the walking surface (ground).

\begin{tabular}{ccc}
\hline \hline Joint & \multicolumn{2}{c}{ Motion specifications } \\
\hline \multirow{2}{*}{ Hip } & Rotation & Roll \\
& Flexion/Extension & Pitch \\
Knee & Flexion/Extension & Yaw \\
& Rotation & Pitch \\
Ankle & Flexion/Extension & Roll \\
& Abduction/Adduction & Pitch \\
\hline & & Yaw \\
\hline
\end{tabular}

TABLE I: Activated Degrees of Freedom.

table

The model which describes the dynamic during the single support phase and the model which describes the dynamic when the swing foot impacts the ground are derived using the Newton-Euler method. The vector $q=\left[q_{0}, \ldots, q_{14}\right]^{T}$ (figure 2, left-hand side) describes the shape and the orientation of the biped_during a single support phase where the angle, $q_{0}=$ $\angle\left(x_{s} \equiv\right.$, denote the angle of the rotation of the stance foot about its toe. During the flat-foot sub-phase the stance foot remains flat on the ground, $q_{0}=0$, is now an absolute ancle referenced to the frame $R_{s}\left(x_{s}, \bar{\equiv}\right)$. Thus, the vector of $\overline{\overline{\bar{V}}}$ configuration is reduced to $\left.q \underline{\underline{v}} q_{1}, \ldots, q_{14}\right]^{T}$, (figure 2, righthand side). The torques are grouped into a $14 \times 1$ torque vector $\Gamma=\left[\Gamma_{1}, \ldots, \Gamma_{14}\right]^{T}$. The torque $\Gamma_{i}$ is applied at joint $q_{i}$ for $1 \leq$ $i \leq 14$.

\section{B. Geometric description of the biped}

To define the geometric structure of the biped walking system we used the parametrization nroposed for the manipulator robots. We considered only $\overline{\bar{\nu}}$ tric cyclic walking thus the definition of the complete motion can be reduced to the definition of the motion for one half step. Since the toe of the right foot (stance foot) is in support during all the studied half step, the reference link is the ground and the supporting foot is connected to the reference link by a rotating joint $q_{0}$. The link

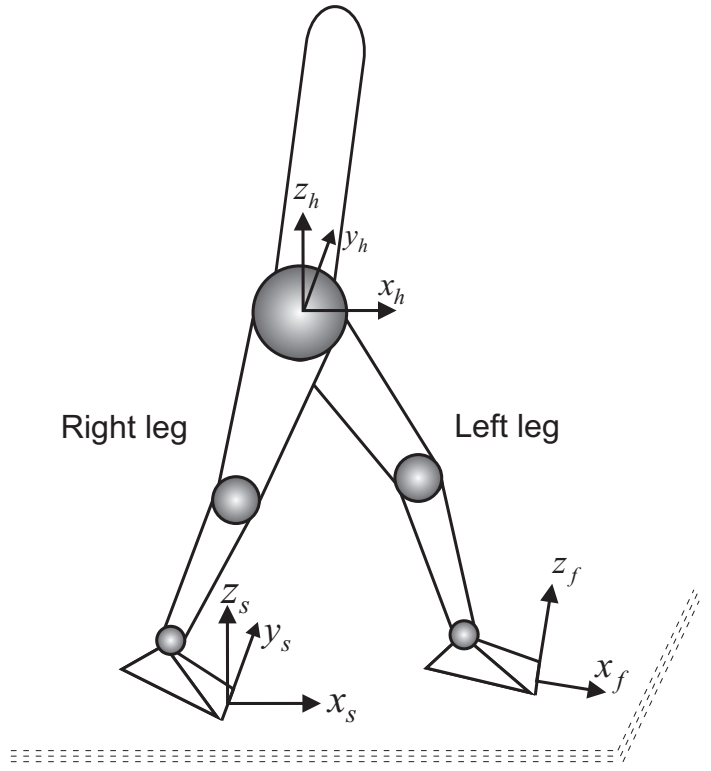

Fig. 1: The studied 3D biped, the Cartesian coordinates are indicated at the middle of the hips and the toe of the feet with respect to the reference frame, $R_{S}\left(x_{s}, y_{s}, z_{s}\right)$, attached to the sole of the foot. figure $\overline{\bar{\nu}}$

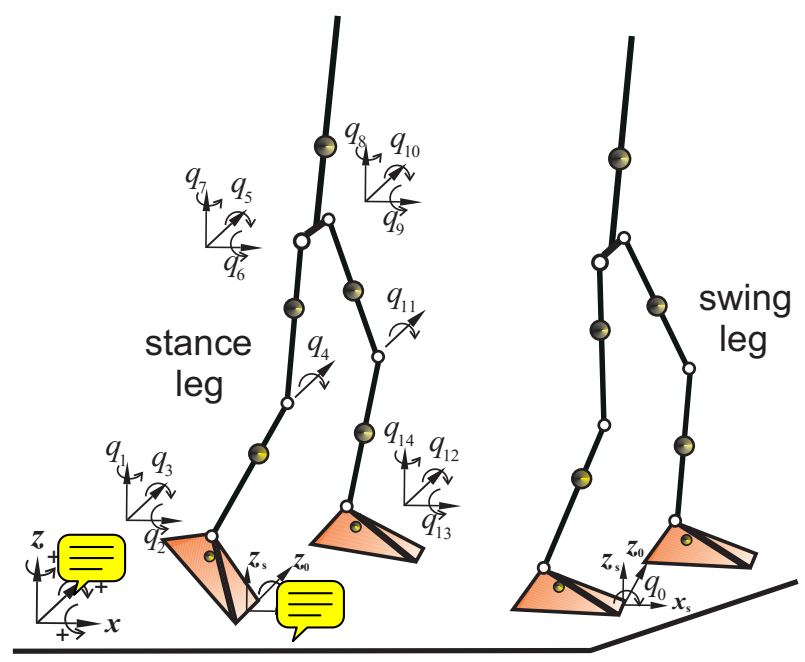

Fig. 2: Model of the studied biped with coordinate convention. In foot rotation sub-phase, $q_{0}$, denotes a relative angle, while in flatfoot sub-phase $q_{0}=0$ is an absolute angle.

figure

seven (swing foot) is considered the terminal link. Therefore we have a simple open loop robot which geometric structure can be described using the notation of Khalil and Kleinfinger [13]. The definition of the link frames is presented in figure 3 and the corresponding geometric parameters are given in Table II. The frame $R_{s}$ is fixed to the tip of the right foot to form a right-handed coordinate frame. The frame to the tip of the left foot in the same way as $R_{s}$. Each foot is determined by the width $l_{p}$ and the length $L_{p}$.

\section{Dynamic modelling}

The gait is composed of stance phases. This stance phase can be composed of a flat-foot phase only or two sub-phases: 


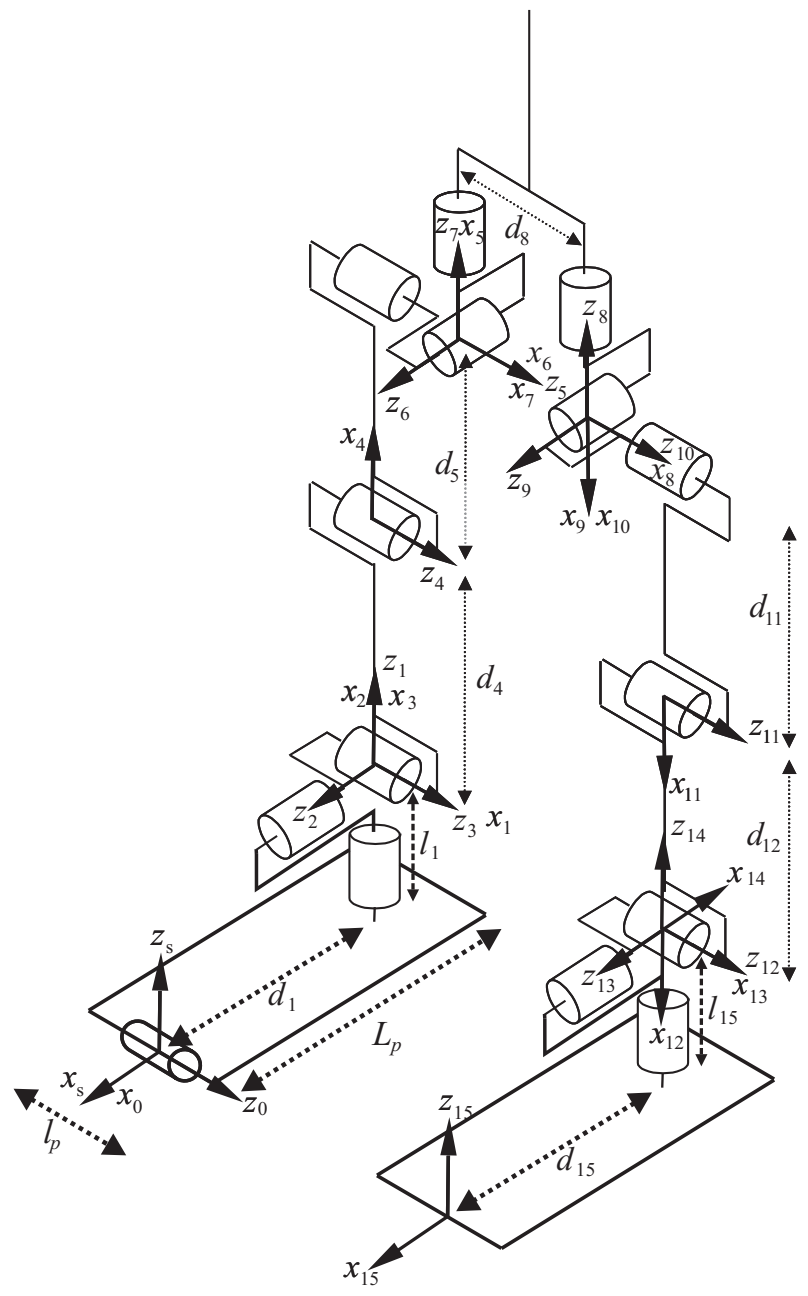

Fig. 3: The multi-body model and link frames of the biped robot. figure

a flat-foot sub-phase and a foot rotation sub-phase. A passive impact exists at the end of the half step, the impacting foot is assumed to be flat on the ground. The biped dynamic models of the phase where the stance foot is flat on the ground, where the stance foot rotates about the stance toe, and the impact model are derived.

1) The single support phase model: flat-foot sub-phase: During this sub-phase, the stance foot is assumed to remain in flat contact on the horizontal ground, i.e., no sliding motion, no take-off, no rotation. Therefore the configuration of the robot is described by only fourteen coordinates. Let $q \in \mathbb{R}^{14}$ be the generalized coordinates, where $q_{1}, \ldots, q_{14}$ denote the relative angles of the joints, $\dot{q} \in \mathbb{R}^{14}$ and $\ddot{q} \in \mathbb{R}^{14}$ are the velocity vector and the acceleration vector respectively. The dynamic model is easily obtained with the method of Newton-Euler [14], which must be adapted to determine the ground wrench. Thus, the model is written in the form

$$
\left[\begin{array}{c}
R_{F} \\
\Gamma
\end{array}\right]=\mathrm{f}\left(q, \dot{q}, \ddot{q}, F_{t}\right)
$$

where $\Gamma \in \mathbb{R}^{14}$ is the joint torque vector, $R_{F}=\left[\mathbf{f}_{R}, \mathbf{m}_{R}\right]^{T}$ is the ground reaction wrench on the stance foot and $F_{t}$ represents the external wrenches (forces and torques), exerted on link 0 to the terminal link. In single support phase we assume that

\begin{tabular}{|c|c|c|c|c|c|}
\hline$j$ & $a(j)$ & $\alpha_{j}$ & $\theta_{j}$ & $r_{j}$ & $d_{j}$ \\
\hline 0 & -1 & $-\frac{\pi}{2}$ & $q_{0}$ & 0 & 0 \\
1 & 0 & $\frac{\pi}{2}$ & $q_{1}+\frac{\pi}{2}$ & $l_{1}$ & $d_{1}$ \\
2 & 1 & $\frac{\pi}{2}$ & $q_{2}+\frac{\pi}{2}$ & 0 & 0 \\
3 & 2 & $\frac{\pi}{2}$ & $q_{3}$ & 0 & 0 \\
4 & 3 & 0 & $q_{4}$ & 0 & $d_{4}$ \\
5 & 4 & 0 & $q_{5}$ & 0 & $d_{5}$ \\
6 & 5 & $-\frac{\pi}{2}$ & $q_{6}-\frac{\pi}{2}$ & 0 & 0 \\
7 & 6 & $-\frac{\pi}{2}$ & $q_{7}$ & 0 & 0 \\
8 & 7 & 0 & $q_{8}$ & 0 & $d_{8}$ \\
9 & 8 & $\frac{\pi}{2}$ & $q_{9}-\frac{\pi}{2}$ & 0 & 0 \\
10 & 9 & $-\frac{\pi}{2}$ & $q_{10}$ & 0 & 0 \\
11 & 10 & 0 & $q_{11}$ & 0 & $d_{11}=d_{5}$ \\
12 & 11 & 0 & $q_{12}$ & 0 & $d_{12}=d_{4}$ \\
13 & 12 & $\frac{\pi}{2}$ & $q_{13}+\frac{\pi}{2}$ & 0 & 0 \\
14 & 13 & $-\frac{\pi}{2}$ & $q_{14}+\frac{\pi}{2}$ & 0 & 0 \\
15 & 14 & 0 & $\pi$ & $l_{15}=-l_{1}$ & $d_{15}=d_{1}$ \\
\hline
\end{tabular}

TABLE II: Geometric parameters of the biped. table<smiles>[C+]1=CC=CC=1</smiles>

$F_{t}=0$. Note that this sub-phase exists under the assumption that the zero moment point remains inside the convex hull of the foot support region.

2) Newton-Euler algorithm: The Newton-Euler method permits to calculate the dynamic model as defined in equation (1). This method proposed by Luh, Walker et Paul [15] is based on tw $=$ ursive calculations. Associated with our choice of

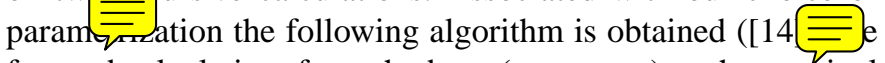
forward calculation, from the base (stance toe) to the terminal link (swing toe) determines the velocity, the accelerations and total forces and moments on each link. Then the backward calculations, from swing toe to stance toe, gives the joint torques and reaction forces using equation of equilibrium of each link successively.

\section{Forward recursive equations}

For each link $j$ with its associated frame $R_{j}$, and considering the link $j-1$ as its antecedent, its angular velocity ${ }^{j} \omega_{j}$, and the linear velocity ${ }^{j} \mathbf{V}_{j}$ of the origin $O_{j}$ of $R_{j}$ are

$$
\begin{aligned}
{ }^{j} \omega_{j}= & { }^{j} \omega_{j-1}+\bar{\sigma}_{j} \dot{q}_{j}{ }^{j} \mathbf{a}_{j} \\
{ }^{j} \mathbf{V}_{j}= & { }^{j} \mathbf{A}_{j-1}\left({ }^{j-1} \mathbf{V}_{j-1}+{ }^{j-1} \omega_{j-1} \times{ }^{j-1} \mathbf{P}_{j}\right) \ldots \\
& +\sigma_{j} \dot{q}_{j}{ }^{j} \mathbf{a}_{j}
\end{aligned}
$$

with ${ }^{j} \mathbf{A}_{j-1}$, the orientation matrix of the frame $R_{j-1}$ in the frame $R_{j}, \sigma_{j}=0$ when the $j$ joint is a revolute joint, $\sigma_{j}=1$ when the $j$ joint is prismatic joint and $\bar{\sigma}_{j}=1-\sigma_{j},{ }^{j} \mathbf{a}_{j}$ is an unit vector along the $z_{j}$ axis, ${ }^{j-1} \mathbf{P}_{j}$ is the vector expressing the origin of frame $R_{j}$ in frame $R_{j-1}$. The angular acceleration of link $j$ and the linear acceleration of the origin $O_{j}$ of $R_{j}$ are

$$
\begin{aligned}
{ }^{j} \dot{\boldsymbol{\omega}}_{j}= & { }^{j} \mathbf{A}_{j-1}^{j-1} \dot{\boldsymbol{\omega}}_{j-1}+\overline{\boldsymbol{\sigma}}\left(\ddot{q}_{j}{ }^{j} \mathbf{a}_{j}+{ }^{j} \omega_{j-1} \times \dot{q}_{j}^{j} \mathbf{a}_{j}\right) \\
{ }^{j} \dot{\mathbf{V}}_{j}= & { }^{j} \mathbf{A}_{j-1}\left({ }^{j-1} \dot{\mathbf{V}}_{j-1}+{ }^{j-1} \mathbf{U}_{j-1}{ }^{j-1} \mathbf{P}_{j}\right) \ldots \\
& +\boldsymbol{\sigma}_{j}\left(\ddot{q}_{j}{ }^{j} \mathbf{a}_{j}+2^{j} \omega_{j-1} \times \dot{q}_{j}^{j} \mathbf{a}_{j}\right)
\end{aligned}
$$

where ${ }^{j} \mathbf{U}_{j}={ }^{j} \hat{\boldsymbol{\omega}}_{j}+{ }^{j} \hat{\omega}_{j}{ }^{j} \hat{\boldsymbol{\omega}}_{j}$. Matrices ${ }^{j} \hat{\boldsymbol{\omega}}_{j} \in \mathbb{R}^{3 \times 3}$ and ${ }^{j} \hat{\omega}_{j}$ $\in \mathbb{R}^{3 \times 3}$ designate the skew matrices associated with the vectors ${ }^{j} \dot{\omega}_{j}$ and ${ }^{j} \omega_{j}$ respectively. 


$$
\hat{\omega}=\left[\begin{array}{ccc}
0 & -\omega_{z} & \omega_{y} \\
\omega_{z} & 0 & -\omega_{x} \\
-\omega_{y} & \omega_{x} & 0
\end{array}\right]
$$

The total inertial forces and moments for link $j$ are

$$
\begin{aligned}
{ }^{j} \mathbf{F}_{j} & =M_{j}{ }^{j} \dot{\mathbf{V}}_{j}+{ }^{j} \mathbf{U}_{j}{ }^{j} \mathbf{M} \mathbf{S}_{j} \\
{ }^{j} \mathbf{M}_{j} & ={ }^{j} \mathbf{J}_{j}{ }^{j} \dot{\omega}_{j}+{ }^{j} \omega_{j} \times\left({ }^{j} \mathbf{J}_{j}{ }^{j} \omega_{j}\right)+{ }^{j} \mathbf{M} \mathbf{S}_{j} \times{ }^{j} \dot{\mathbf{V}}_{j}
\end{aligned}
$$

with ${ }^{j} \mathbf{J}_{j}$ inertia tensor of link $j$ with respect to $R_{j}$ frame, ${ }^{j} \mathbf{M S}_{j}$ is the first moments vector of link $j$ around the origin of $R_{j}$ frame and $M_{j}$ the mass of the link $j$. The anteced ink to the link $\theta$ (stance foet) is not defined. For the iteration of the stance foot, only the equations (4) - (7) are used.

The initial conditions are

$$
\omega_{0}=\mathbf{0}, \quad \dot{\omega}_{0}=\mathbf{0} \text { and } \dot{V}_{0}=\dot{V}_{0}-\mathbf{g}
$$

with $\mathbf{g}$ the acceleration due to gravity to take into account the gravity effect, and $\dot{V}_{0}=\mathbf{0}$ is the real acceleration of the frame $R_{0}$.

Note that if the stance foot rotates about its toe the initial conditions are; $\omega_{0}=\dot{q}_{0} \vec{y}_{s}$ and $\dot{V}_{0}=\mathbf{0}$.

\section{Backward recursive equations}

The backward recursive equations are given as, for $j=$ $14, \ldots, 0$

$$
\begin{aligned}
{ }^{j} \mathbf{f}_{j} & ={ }^{j} \mathbf{F}_{j}+{ }^{j} \mathbf{f}_{j+1} \\
{ }^{j-1} \mathbf{f}_{j} & ={ }^{j-1} \mathbf{A}_{j}{ }^{j} \mathbf{f}_{j} \\
{ }^{j} \mathbf{m}_{j} & ={ }^{j} \mathbf{M}_{j}+{ }^{j} \mathbf{A}_{j+1}{ }^{j+1} \mathbf{m}_{j+1}+{ }^{j} \mathbf{P}_{j+1} \times{ }^{j} \mathbf{f}_{j+1}
\end{aligned}
$$

These recursive equations will be initialized by the forces and moments exerced on the swing toe by the environment ${ }^{j} \mathbf{f}_{j+1}$ and ${ }^{j} \mathbf{m}_{j+1}$. In single support ${ }^{j} \mathbf{f}_{j+1}=0,{ }^{j} \mathbf{m}_{j+1}=0$. When $j=0,{ }^{0} \mathbf{f}_{0}$ and ${ }^{0} \mathbf{m}_{0}$ are the force exerted on the stance foot, i.e., the ground reaction force and moment rewritten as ${ }^{s} \mathbf{f}_{R}$ and ${ }^{s} \mathbf{m}_{R}$ expressed in the frame $R_{s}$.

If we neglect the friction and the motor inertia effects, the torque (or the force) $\Gamma_{j}$, is obtained by projecting $m_{j}$ (or $\mathbf{f}_{j}$ ) along the joint axis $\left(z_{j}\right)$

$$
\Gamma_{j}=\left(\sigma_{j}{ }^{j} \mathbf{f}_{j}+\bar{\sigma}_{j}{ }^{j} \mathbf{m}_{j}\right)^{T}{ }^{j} \mathbf{a}_{j}
$$

$\Gamma_{0}$ is not defined, since there is no actuator.

3) Flat-foot sub-phase, the ZMP condition: The ground reaction wrench is known in the reference frame $R_{S}$. This reference frame is associated with the stance foot. The position of the ZMP (Zero Moment Point) defined as the point of the sole such that the moment exerted by the ground is zero along the axis $x_{s}$ and $y_{s}$ is such that:

$$
x_{Z M P}=\frac{-{ }^{s} m_{R y}}{{ }^{s} f_{R z}} \text { and } y_{Z M P}=\frac{{ }^{s} m_{R x}}{{ }^{s} f_{R z}} .
$$

The flat foot phase exists only if the foot does not rotate, then for a rectangular foot the $Z M P$ must satisfy:

$$
\frac{-l_{p}}{2} \leq \frac{{ }^{s} m_{R x}}{{ }^{s} f_{R z}} \leq \frac{l_{p}}{2} \quad \text { and } \quad-L_{p} \leq \frac{-{ }^{s} m_{R y}}{{ }^{s} f_{R z}} \leq 0,
$$

where $l_{p}$ is the width and $L_{p}$ is the length of the feet. Because of the stance foot is flat on the ground, the ZMP is equivalent to the $\operatorname{CoP}$ (Center of Pressure) (see [16], [17], [18]).

4) Foot rotation sub-phase, angular momentum about the toe: In this sub-phase the stance heel of the robot rises from the ground and the robot begins to roll over the stance toe. Thus the variable, $q_{0}=\angle\left(x_{s}, x_{0}\right)_{z_{0}}$, is added.

Let $q_{r}=\left[q_{0} ; q\right] \in \mathbb{R}^{15}$ be the generalized coordinates, $\dot{q}_{r} \in$ $\mathbb{R}^{15}$ and $\ddot{q}_{r} \in \mathbb{R}^{15}$ are the velocity vector and the acceleration vector respectively. The dynamic model is obtained from

$$
\left[\begin{array}{c}
R_{F} \\
\Gamma
\end{array}\right]=\mathrm{f}\left(q_{r}, \dot{q}_{r}, \ddot{q}_{r}, F_{t}\right)
$$

where $\Gamma \in \mathbb{R}^{14}$ is the joint torque vector. Since only 14 torques are applied and 15 variables $q_{r}$ describe the biped configuration, the dynamic model is under-actuated.

The fact that the stance foot rotates about its toe and there is no actuation between the stance toe and the ground, the $C o P$ is forced to remain strictly of the front limit of the stance foot. In order to satisfy this condition, the position of $C o P$ is imposed. Therefore the ground reaction wrench, $R_{F}$, on the stance foot is rewritten as

$$
R_{F}=\left[{ }^{s} f_{R x},{ }^{s} f_{R y},{ }^{s} f_{R z},{ }^{s} m_{R x},{ }^{s} m_{R y},{ }^{s} m_{R z}\right]^{T}
$$

with the ground reaction moment about $y_{s}$, expre $\overline{\overline{\overline{\bar{F}_{n}}}}$ the frame $R_{s},{ }^{s} m_{R y}=0$.

From the fact that $q_{0}$ defines only the orientation of the biped as a rigid body rotating about its toe, the angular momentum $\sigma_{y s}$ about axis $y_{s}$ is denoted by:

$$
\sigma_{y s}=m_{t}\left(\dot{x}_{c m} z_{c m}-x_{c m} \dot{z}_{c m}\right)
$$

where $m_{t}$ is the mass of the biped, $x_{c m}$ and $z_{c m}$ are the horizontal and vertical positions of the center of mass of the biped and $\dot{x}_{c m}$ and $\dot{z}_{c m}$ are the velocities respectively, measured with respect to frame $R_{s}$. Now, using the angular momentum theorem, and from the rotational dynamic equilibrium of the biped as a rigid body, the rate of change of the angular momentum of the biped about its toe is:

$$
\dot{\sigma}_{y s}={ }^{s} m_{R y}+m_{t} g x_{c m},
$$

since ${ }^{s} m_{R y}=0$, this equation is rewritten as

$$
\dot{\sigma}_{y_{s}}=m_{t} g x_{c m},
$$

which describes the external applied torque, where $g$ is the acceleration due to gravity. For a motion $q_{r}, \dot{q}_{r}, \ddot{q}_{r}$, satisfy (18), the condition describing the under-actuation is sticied, the torque $\Gamma$ and reaction force can be calculated $\overline{\bar{\nu}}_{4}$ ). When the supporting foot is in rotation about the toe, in order to maintain the balance in dynamic walking the $C o P$ must be remain on the lateral axis bounded by $l_{p}$, then:

$$
\frac{-l_{p}}{2} \leq \frac{{ }^{s} m_{R x}}{{ }^{s} f_{R z}} \leq \frac{l_{p}}{2}
$$




\section{IMPACT MODEL}

An impact occurs at the end of the single support ppace when the swing foot contacts the ground. This impack assumed to be instantaneous and inelastic, i.e., the velocity of the swing foot touching the ground is zero after its impact. We assume that the ground reaction at the instant of the impact is described by a Dirac delta-function with intensity $I_{w f}$. The previous stance foot is motionless before the impact and not remains on the ground after the impact.

Under these hypothesis, the solution of the impact consists to determine the velocity after the impact and the impulsive forces, by considering known the velocity before the impact. Let us introduce the generalized coordinates as; $X=$ $\left[X_{0}, \alpha_{0}, q\right]^{T} \in \mathbb{R}^{20}$, where $X_{0}$ and $\alpha_{0}$ are the position and the orientation variables of frame $R_{0}$. The robot velocity is $V=\left[{ }^{s} V_{0},{ }^{s} \omega_{0}, \dot{q}\right]^{T} \in \mathbb{R}^{20}$, with ${ }^{s} \omega_{0_{y}}=\dot{q}_{0}$ and the robot acceleration is $\dot{V}=\left[{ }^{s} \dot{V}_{0},{ }^{s} \dot{\omega}_{0}, \ddot{q}\right]^{T} \in \mathbb{R}^{20}$, with ${ }^{s} \dot{\omega}_{0_{y}}=\ddot{q}_{0}$. Using these generalized coordinates the dynamic model in double support, under the Lagrange form, can be written as:

$$
D(X) \dot{V}+C(V, q)+G(X)+D_{J} F_{w f}=\left[\begin{array}{c}
R_{F} \\
\Gamma
\end{array}\right]
$$

where $F_{w f}$ represents the vector of ground reaction forces and moments on the swing foot, $D \in \mathbb{R}^{20 \times 20}$ is the symmetric definite positive inertia matrix, $C \in \mathbb{R}^{20}$ represents the Coriolis and centrifugal forces, $G \in \mathbb{R}^{20}$ is the vector of gravity, $D_{\perp} \in$ $\mathbb{R}^{20 \times 6}$ is the Jacobian matrix of the robot. These matri computed using the Newton-Euler algorithm (see appendix).

The model of impact can be obtained by integrating (20) over the du $\overline{\overline{\bar{\gamma}}}$ of the impact. The torques provided by the actuators at the joints, Corilois and gravity forces have finite value, thus they do not influence the impact. Therefore, because of the fact that the stance foot take-off the ground after the impact, the impulsive ground reaction on this foot must be null. Consequently the impact equations can be written as:

$$
D(X(T)) \Delta V=-D_{J} I_{w f}
$$

where $I_{w f}$ is the intensity of Dirac delta-fu $\overline{\overline{\overline{ }}}$ for the impulsive contact force [19]. $\Delta V$ is the variation of velocity at the impact, $\Delta V=V^{+}-V^{-}$, where $V^{-}$is the velocity of the robot before impact and $V^{+}$its velocity after impact. $X(T)$ denotes the configuration of the robot at instant of the impact, which does not present instantaneous change. The swing foot after the impact becomes the stance foot. Therefore, its velocity becomes zero after the impact, which may be written as:

$$
\begin{aligned}
D_{J}^{T} V^{+} & =0 \\
{\left[\begin{array}{c}
{ }^{s} V_{0}^{-} \\
{ }^{s} w_{0}^{-}
\end{array}\right] } & =\left[\begin{array}{c}
0_{3 \times 1} \\
\dot{q}_{0} \vec{y}_{s}
\end{array}\right]
\end{aligned}
$$

The combined set of equations (21) a 2 2) form the systems of equations

$$
\left[\begin{array}{cc}
D(X(T)) & D_{J} \\
D_{J}^{T} & 0_{6 \times 6}
\end{array}\right]\left[\begin{array}{c}
V^{+} \\
I_{w f}
\end{array}\right]=\left[\begin{array}{c}
D(X(T)) V^{-} \\
0_{6 \times 1}
\end{array}\right]
$$

Solving (24) yields

$$
\left[\begin{array}{c}
V^{+} \\
I_{w f}
\end{array}\right]=\left[\begin{array}{c}
\Delta_{v} \\
\Delta_{I_{w f}}
\end{array}\right] V^{-}
$$

where,

$$
\Delta_{I_{w f}}=\left(D_{J}^{T} D^{-1} D_{J}\right)^{-1} D_{J}^{T}
$$

and

$$
\Delta_{v}=-D^{-1} D_{J} \Delta_{I_{w f}}+\overline{\overline{\bar{\nu}}}
$$

From the hypotesis that the previous supporting foot takes off, the contact conditions on this foot do not directly affect the impact equation, the model is independent on the fact that the impact occurs at the end of the flat-foot sub-phase or at the end of the foot rotation sub-phase.

In order to validate the impact model, it $\mathrm{\equiv} \equiv$ verified that the impulsive force must be directed upward and be inside the friction cone. To ensure a take-off of stance foot, the vertical velocity component of foot tip must be positive. The equilibrium of the foot at the impact allows to determine the position of the ZMP. This constraint is developed in [20].

\section{GAit OPTIMIZATION FOR THE CYCLIC WALKING}

\section{A. Gait without foot rotation}

1) The optimization parameters: The biped is driven by 14 torques, and its configuration is given in single support phase by 14 coordinates $q$. To transform the optimization problem into a finite dimension problem, the joint motion is described as a parametric function. We choose a polynomial function of time. $q(0), q\left(T_{f}\right)$ are the initial and final configurations of the single support phase, respectively, $T_{f}$ is the duration of this phase.

To insure continuity between two successive half steps, the position and velocity of the biped at the beginning and end of each phase must be taken into account by the parameters of the polynomial functions. So, third-order polynomial functions are needed. Thus each of the fourteen joint variables is defined by a third-order polynomial,

$$
q_{k}(t)=\sum_{j=0}^{3} a_{k j}(t)^{j}, \quad k=1, \ldots, 14 \quad \forall t \in\left[0, T_{f}\right]
$$

where $k$ is the joint number. The polynomial functions $q_{k}(t), k=1, \ldots, 14$ are uniquely defined by $q_{i}, q_{f}, \dot{q}_{i}, \dot{q}_{f}$. The indices $i$ and $f$ correspond to the initial (at $t=0$ ) and final (at $t=T_{f}$ ) configuration ${ }^{1}$ of the robot, respectively.

In fact, the initial and final configurations for the stance phase are double support configurations with the two feet flat on the ground. Thus only 8 independent variables are necessary to define the initial and final configurations of the biped legs. We use the twist motion of the swing foot denoted by $\psi_{f}$ and its position $\left(x_{f}, y_{f}\right)$ in the horizontal plane as well as the situation of the middle of the hips defined

\footnotetext{
${ }^{1}$ To avoid any collision of the swing foot with the ground, one def intermediate configuration at $t=T_{f} / 2$. In such case, the joint motion will be described by a cubic spline function.
} 
by $\left(x_{h}, y_{h}, z_{h}, \theta_{h}, \phi_{h}\right)$ where $\theta_{h}$ and $\phi_{h}$ are the inclination in the sagittal plane and rotation about axis $z_{h}$ of the torso. The desired trajectory has the particularity to be cyclic: two following half steps must be identical and, more precisely, the legs will swap their roles from one half step to the next. The condition of periodicity is used to define the trajectory only on one half step to reduce the number of optimization parameters. In this way, we avoid to use two single support models.

Since the position of the robot is constant during the passive impact (touch down configuration) and since the legs swap their roles from one half step to the next, the generalized coordinates must be relabeled as a matrix $E$ :

$$
q_{i}=E q_{f}
$$

where, $E_{14 \times 14}$ describes an anti-diagonal identity matrix

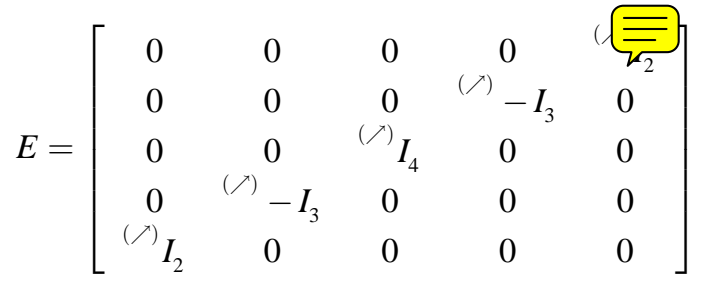

The final configuration $q_{f}$ is determined from the inverse kinematics solution of each leg.

The velocity of the robot after the impact can be defined as function of the velocity before the impact. Then, from (25), the last fourteen rows of $V^{+}$should be used to obtain the ini $\overline{\overline{\bar{\nu}}}$ relocity $\dot{q}_{i}$ by:

$$
\dot{q}_{i}=E V_{(7: 20)}^{+}
$$

Using (29) and (31), the polynomial functions $q(t)$, can be defined as function of $T_{f}, \dot{q}_{f}, x_{f}, y_{f}, \psi_{f}, x_{h}, y_{h}, z_{h}, \theta_{h}, \phi_{h}$. The optimal trajectory is defined by 23 parameters only.

2) Torque and forces: When function $q(t)$ is chosen, the joint velocity and the joint acceleration can be deduced by the derivation of the polynomial function. The dynamic model (1) gives the torques required to produce the motion and the reaction force.

\section{B. Walk with foot rotation}

1) The flat-foot sub-phase: When a sub-phase with foot rotation is added, the optimization process is modified. The two sub-phases are $\bar{\equiv}$ ribed separately and the condition of continuity in configuration and velocity between the subphases are added. For each sub-phase, the final state of the biped is chosen to be defined from the optimization variables, the initial state is deduced from the continuity conditions.

The flat-foot sub-phase is described as previously. The difference is only that the final configuration for this phase is not a double support configuration but a single support with flat stance foot configuration, thus 14 variables are used to describe this configuration $\left(q_{f}\right)$, an 2 optimization variables describe this sub-phase, $T_{f}, q_{f}$ and
2) The foot-rotation sub-phase: During the foot rotation sub-phase, the biped is driven by 14 torques, and its configuration is given in single support phase by 15 coordinates $q_{r}$. Thus the biped is under-actuated and its motion cannot be freely chosen. Studies of control of such an under-actuated robot [11], [12] have shown that a geometric evolution of the robot $q_{r}(s)$ can be chosen. For a given function $q_{r}(s)$ within some limits, function $s(t)$ corresponding to a motion compatible with the dynamic model can be deduced using (17). In the optimization process, the joint evolution is described by function $q_{r}(s)$. This way solves the under-actuation problem and avoids the use of equality constraints as in [21], this point is detailed in IV-B.3.

We choose to define the evolution of the joint variables as a polynomial of $s$, where $s$ is a monotonic function from 0 to $1 . q_{r}(0), q_{r}(1)$ are the initial and final configurations of the foot rotation support phase, respectively. Then,

$$
q_{r k}(s)=\sum_{j=0}^{3} a_{k j}(s)^{j}, \quad k=0, \ldots, 14 \quad \forall s \in[0,1]
$$

where $k$ is the joint number. The polynomial functions $q_{r k}(s), k=0, \ldots, 14$ are uniquely defined by $q_{i r}, q_{f r}, \frac{d q_{i r}}{d s}, \frac{d q_{f r}}{d s}$. The indices $i r, f r$ correspond to the initial (at $s=0$ ), final (at $s=1)$ state of the robot for this sub-phase, respectively. The velocity of the robot is $\dot{q}_{r}=\frac{d q_{r}}{d s} \dot{s}$. Since $\dot{s}$ is not given, only the direction of the joint velocity is given, not its amplitude.

In fact, the initial state for this sub-phase are the final state for the flat-foot sub-phase $q_{i r}=\left[\left(q_{f 0}=0\right) ; q_{f}\right]$, thus there are known by the 29 optimization parameters for the flat-foot subphase. The initial velocity of the robot is known, $\dot{q}_{i r}=\left[\left(\dot{q}_{f 0}=\right.\right.$ $\left.0) ; \dot{q}_{f}\right]$. The initial vector $\frac{d q_{i r}}{d s}$ can be deduced if $\dot{s}(0)$ is known, this term will be an optimization variable.

The final configuration is a double support configuration with only one foot flat on the ground, thus 9 coordinates are used to define this configuration $x_{f}, y_{f}, \psi_{f}, x_{h}, y_{h}, z_{h}, \theta_{h}, \phi_{h}, q_{f 0}$. The joint path $q_{r}(s)$ during the foot rotation sub-phase can be calculated with 25 optimization variables : 9 for $q_{r f}, 15$ for $\frac{d q_{r f}}{d s}$ and $\dot{s}(0)$.

3) From joint trajectories to joint motions: The joint evolution is given as $q_{r}(s)$, but since the robot is under-actuated, function $s(t)$ must be such that the robot motion satisfies (17). Because $\dot{q}_{r}(s)$ is proportional to $\dot{s}$, the angular momentum

$$
\sigma_{y s}=m_{t}\left(\dot{x}_{c m} z_{c m}-x_{c m} \dot{z}_{c m}\right),
$$

can be rewritten as:

$$
\begin{gathered}
\sigma=I(s) \dot{s}(s) \\
\dot{\sigma}=\operatorname{mgx}_{c m}\left(q_{r}(s)\right)
\end{gathered}
$$

These two equations can be combined to have for $0 \leq s \leq 1$ [12]:

$$
\begin{gathered}
\frac{1}{2} I(0)^{2} \dot{s}(0)^{2}=\frac{1}{2} I(s)^{2} \dot{s}(s)^{2}+V(s) \\
V(s)=-m g \int_{0}^{s} I(\xi) x_{g}(\xi) d \xi
\end{gathered}
$$

Since function $I(s)$ and $V(s)$ can be calculated for any given function $q_{r}(s)$, it follows that the initial value $\dot{s}(0)$ permits to 
define the function $\dot{s}$ completely and thus $s(t)$.

$$
\dot{s}(s)=\sqrt{\frac{I(0)^{2} \dot{s}(0)^{2}-2 V(s)}{I(s)^{2}}}
$$

Polynomials $q_{r}(s)$ is defined with the assumption that $s$ is a well defined increasing function, thus the following conditions must be satisfied:

$$
\begin{array}{ccc}
\dot{s}(0) \quad> & \sqrt{\frac{2 \max _{s}(V(s))}{I(0)^{2}}} \\
I(s) \neq 0 \quad \text { for } & 0 \leq s \leq 1
\end{array}
$$

These constraints are taken into account in the optimization process.

The value of $\dot{s}$ at the end of the foot rotation sub-phase can be deduced from (36), thus the velocity of the robot at the end of the foot rotation sub-phase is :

$$
\dot{q}_{r f}=\sqrt{\frac{I(0)^{2} \dot{s}(0)^{2}-2 V(1)}{I(1)^{2}}} \frac{d q_{r f}}{d s}
$$

Since the impact occurs in the configuration $q_{r f}$ with the velocity $\dot{q}_{r f}$, the initial state of the robot for the flat-foot sub-phase can be deduced from equations (29) and (31). The duration of the foot-rotation phase is not a direct optimization variable, it is the result of the integration of the function $\dot{s}(s)$ than defines at which time $s=1$, i.e.,

$$
T_{r}=\int_{0}^{1} \frac{1}{\dot{s}} d s
$$

4) Torque and forces: For the foot rotation sub-phase, when the function $q_{r}(s)$ is chosen, $\dot{s}(s)$ can be calculated by (36). Thus the joint velocity is:

$$
\dot{q}_{r}(s)=\frac{d q_{r}}{d s} \dot{s}(s)
$$

and the joint acceleration can be written as:

$$
\ddot{q}_{r}(s)=\frac{d^{2} q_{r}}{d s^{2}} \dot{s}^{2}+\frac{d q_{r}}{d s} \ddot{s}(s)
$$

In order to deduce $\ddot{s}$, we use the linearity of the torque $\Gamma$ with respect to the acceleration $\ddot{s}$ and the fact that the torque about the toe is zero. Therefore,

$$
\stackrel{\equiv}{\bar{\equiv}=u \ddot{s}+v}
$$

where, from (14) with $F_{t}=0$,

$$
\bar{\Gamma}=\left[\begin{array}{c}
R_{F_{R}} \\
\Gamma
\end{array}\right]=\mathrm{f}\left(q_{r}(s), \dot{q}_{r}(s), \ddot{q}_{r}(s)\right)
$$

Using the Newton-Euler algorithm, $\bar{\Gamma}$ is calculated for $\ddot{s}=0$ and $\ddot{s}=1$; these vectors are denoted by $\bar{\Gamma}^{0}$ and $\bar{\Gamma}^{1}$ respectively. For any $\ddot{s}$ we have:

$$
\bar{\Gamma}=\left(\bar{\Gamma}^{1}-\bar{\Gamma}^{0}\right) \ddot{s}+\bar{\Gamma}^{0}=u \ddot{s}+v
$$

Thus, $v=\bar{\Gamma}^{0}$ and $u=\left(\bar{\Gamma}^{1}-\bar{\Gamma}^{0}\right)$ are obtained.

Then, $\ddot{s}$ is easily obtained from fifth row of (44), because ${ }^{s} m_{R y}=0$, as:

$$
\ddot{s}=-\frac{v_{5}}{u_{5}}
$$

The indix 5 correspond to the fifth row of $v$ and $u$. Then the torques required to produce the motion are computed as:

$$
\Gamma=u_{(7: 20)}\left(-\frac{v_{5}}{u_{5}}\right)+v_{(7: 20)},
$$

and the ground reaction forces as:

$$
R_{F}=u_{(1: 6)}\left(-\frac{v_{5}}{u_{5}}\right)+v_{(1: 6)} .
$$

\section{Optimal WALK}

\section{A. Constraints and limitations}

The objective of this study is to define a feasible optimal trajectory for a given robot with given actuators. Then, in order to insure that such trajectory is possible, many constraints given by physical or others limitations present during the evolution of the gait cycle have to be considered.

1) Magnitude constraints on position, velocities and torque:

- Each actuator has physical limits such that

$$
\left|\Gamma_{i}\right|-\Gamma_{i, \max } \leq 0, \quad \text { for } i=1, \ldots, 14
$$

where $\Gamma_{i, \max }$ denotes the maximum value for each actuator.

$$
\left|\dot{q}_{i}\right|-\dot{q}_{i, \max } \leq 0, \quad \text { for } i=1, \ldots, 14
$$

where $\dot{q}_{i, \max }$ denotes the maximum velocity for each actuator.

- The upper and lower bounds of joints for the configurations during the motion are:

$$
q_{i, \min } \leq q_{i} \leq q_{i, \max }, \quad \text { for } i=1, \ldots, 14
$$

$q_{i, \min }$ and $q_{i, \max }$ respectively stands for the minimum and maximum joint limits.

2) Geometric constraints in double support phase:

- Position and orientation limitations to define the left foot and the middle of the hips situations described in (IV).

$$
P_{f, l} \leq P_{f} \leq P_{f, u} \text { and } P_{h, l} \leq P_{h} \leq P_{h, u}
$$

where $P_{f}=\left[x_{f}, y_{f}, \psi_{f}\right]^{T}$ and $P_{h}=\left[x_{h}, y_{h}, z_{h}, \theta_{h}, \phi_{e} a_{0}\right]^{T}$ denotes the coordinates to define such configurati $\overline{\overline{\bar{v}}}_{f, l}$, $P_{h, l}$ and $P_{f, u}, P_{h, u}$ are lower limit and upper limit of $P_{f}$ and $P_{h}$.

- In order to avoid the internal collision of both feet through the lateral axis the heel and the toe of the left foot must satisfy

$$
y_{\text {heel }} \leq-a \text { and } y_{\text {toe }} \leq-a
$$

with $a>\frac{l_{p}}{2}$ and $l_{p}$ is the width of right foot. 


\section{3) Walking constraints:}

- During the single support phase, the reaction force exerted by the ground on the stance foot as well as impulsive force acting on the swing foot impacting the ground must be directed upward to avoid take-off, and must be inside the friction cone defined by the friction coefficient $\mu$ to avoid the sliding of the biped. This is equivalent to write

$$
\begin{aligned}
\sqrt{R_{F_{x}}^{2}+R_{F_{y}}^{2}} & \leq \mu R_{F_{z}} \\
\sqrt{I_{w f_{x}}^{2}+I_{w f_{y}}^{2}} & \leq \mu I_{w f_{z}}
\end{aligned}
$$

The conditions of no take-off are deduced by

$$
\begin{gathered}
R_{f_{z}} \geq 0 \\
I_{w f_{z}} \geq 0 .
\end{gathered}
$$

- The swing foot must not touch the ground before the prescribed end of the single support phase, then the $z_{t i p}$ position coordinate of the swing foot tip must be greater than a smooth curve of pre-specified ampli $\overline{\overline{\bar{v}}}$

$z_{\text {tip }} \leq f\left(k, A_{z}\right)$, with $f(-d)=f(d)=0 \quad \forall k \in[-d, d]$

where $A_{z}$ denotes maximum height of the swing foot and $d=x_{f}$ is the step lenght.

- In order to maintain the balance in dynamic walking, the Zero Moment Point which is equivalent to the Center of Pressure $(C o P)$, of the stance foot must be sat $\overline{\overline{\bar{\nu}}}$ for the flat-foot sub-phase

$$
\frac{-l_{p}}{2} \leq \frac{{ }^{s} m_{R_{x}}}{{ }^{s} f_{R_{z}}} \leq \frac{l_{p}}{2} \quad \text { and } \quad-L_{p} \leq \frac{-{ }^{s} m_{R_{y}}}{{ }^{s} f_{R_{z}}} \leq 0,
$$

and for the foot rotation sub-phase

$$
\frac{-l_{p}}{2} \leq \frac{{ }^{s} m_{R_{x}}}{{ }^{s} f_{R_{z}}} \leq \frac{l_{p}}{2} .
$$

where $l_{p}$ is the width and $L_{p}$ is the length of the feet.

- Constraint on the existence of the function $s(t)$ is considered to define the polynomials $q_{r}(s)$. This constraint can be simply written as

$$
\begin{array}{cc}
\dot{s}(0) \quad> & \sqrt{\frac{2 \max _{s}(V(s))}{I(0)^{2}}} \\
I(s) \neq 0 \quad \text { for } & 0 \leq s \leq 1
\end{array}
$$

\section{B. Cost function}

In electrical motors and for a cycle of walk, most part of the energy consumption is due to the loss by Joule effect neglecting the friction. Thus the optimized criterion is proportional to this loss of energy. It is defined as the integral of the norm of the torque for a displacement of one meter:

$$
J_{\Gamma}=\frac{1}{d}\left(\int_{0}^{T_{f}} \Gamma(t)^{T} \Gamma(t) d t+\int_{0}^{1} \Gamma(s)^{T} \Gamma(s) \frac{1}{\dot{s}} d s\right)
$$

where $T_{f}$ is the duration of the flat-foot sub-phase of one half step, $d=x_{f}$ is the step length. The total duration of one half step is defined by $T=T_{f}+T_{r}$, with $T_{r}$ obtained of (39).

\section{Optimization problem}

The objective of this optimization procedure will be to select a feasible solution by minimizing the criterion (61), for a given motion velocity of the robot, satisfying constraints (48)-(60). Let $P=\left[P_{1}, P_{2}, \ldots, P_{54}\right]^{T}$ be the optimization parameters, $J_{\Gamma}(P)$ the criterion and $g(p)=\left[g_{1}(p), g_{2}(p), \ldots, g_{l}(p)\right]^{T}$ the inequality constraints to satisfy, the optimization problem can be formally stated as:

$$
\left.\begin{array}{ll}
\text { Minimize } & J_{\Gamma}(P) \\
\text { subject to } & g_{j}(P) \leq 0 \quad j=1, \ldots, l
\end{array}\right\}
$$

This constrained nonlinear optimization problem is solved using the fmincon function from the package Matlab. Thus, this optimization problem, under constraints, is solved numerically.

The main parameters, used in the presented study, for this humanoid robot are given in table V-E. The parameters are defined with respect to reference frame fixed at each body, see Figure 3.

\section{Walk without foot rotation}

The chosen motion velocity for the three-dimensional bipedal robot is $1.2 \mathrm{~m} / \mathrm{s}(4.32 \mathrm{~km} / \mathrm{h})$, which corresponds to an average walking speed. For this motion, the flat-foot presents a twist rotation equals $0.103 \mathrm{rd}$. The optimal walk has the following characteristics: for one half step, the duration $T_{f}$ is $0.29 \mathrm{~s}$, the step length is $0.354 \mathrm{~m}$. The value of the torque cost criterion $C$ is $11745.23 \mathrm{~N}^{2} \mathrm{~ms}$.

Figure 4 presents the stick-diagram of one step of an optimal walk. Figure 5 shows the validity of nonsliding (53) and no take-off (55) constraints. The Coulomb friction coefficient $\mu$ is $3 / 4$. The average vertical reaction force is $401.7 \mathrm{~N}$, which is coherent with the weight of the biped with mace equals $40.75 \mathrm{~kg}$. For this stable gait, the evolution of $\mathrm{C} \overline{\overline{\bar{\gamma}}}$ is illustrated in figure 6 . This trajectory is the result of the optimization process which evolution remains within the foot area, satisfying (58). The applied torques are shown in figure 8. Note that the torques on the stance leg are higher than on the swing leg, the highest torques concern the hip and the knee. The figure 7 sho $\bar{\equiv}$ position and velocity states of the robot, walking at $1.2 \mathrm{~m} / \mathrm{s}$.

\section{E. Walk with foot rotation}

For purpd $\overline{\overline{\bar{\nu}}}$ fhow that the use of a foot rotation subphase during the single support phase reduces the functional cost, the chosen motion velocity for this simulation is 1.3 $\mathrm{m} / \mathrm{s}(4.68 \mathrm{~km} / \mathrm{h})$. This motion velocity is such that a gait with foot rotation is more efficient than a gait without foot rotation. During the evolution of this motion, the foot in rotation finishes with an angle equals to $0.435 \mathrm{rd}$ and a twist rotation equals $0.101 \mathrm{rd}$. The optimal walk has the following characteristics: for one half step, the duration of the flat-foot and foot rotation sub-phases is $T_{f}=0.130 \mathrm{~s}$ and $T_{r}=0.202$ $\mathrm{s}$, respectively. The step length is $0.433 \mathrm{~m}$. The value of the torque cost criterion $C$ is $3693.98 \mathrm{~N}^{2} \mathrm{~ms}$. 


\begin{tabular}{|c|c|c|c|c|c|c|c|c|}
\hline & & \multicolumn{4}{|c|}{ Parameters of the biped for the right leg } & \multicolumn{3}{|c|}{ Parameters of the biped for the left leg } \\
\hline \multicolumn{2}{|l|}{ Body } & Foot & Femur & Tibia & Torso & Foot & Femur & Tibia \\
\hline \multicolumn{2}{|l|}{ Frame } & $s$ & 3 & 4 & 7 & 14 & 11 & 10 \\
\hline \multicolumn{2}{|l|}{ Mass $(K g)$} & 0.678 & 2.188 & 5.025 & 24.97 & 0.678 & 2.188 & 5.025 \\
\hline \multirow{6}{*}{ Inertia $\left(\mathrm{kg} \cdot \mathrm{m}^{2}\right)$} & $J_{X X}$ & 0.0012618 & 0.001905 & 0.06645 & 3.0115 & 0.00091027 & 0.001905 & 0.06645 \\
\hline & $J_{X Y}$ & 0 & 0 & 0 & -0.0044581 & 0 & 0 & 0 \\
\hline & $J_{X Z}$ & 0 & $\stackrel{n}{=}$ & 0 & -0.67669 & -0.000539 & 0 & 0 \\
\hline & $J_{Y Y}$ & 0 & $0.0 \overline{\bar{\tau}} 6$ & 0.20922 & 3.3357 & 0.002858 & 0.13689 & 0.31733 \\
\hline & $J_{Y Z}$ & 0 & 0 & 0 & -0.013753 & 0 & 0 & 0 \\
\hline & $J_{Z Z}$ & 0 & 0 & 0 & 0.43827 & 0.0026717 & 0.13689 & 0.31733 \\
\hline \multirow{3}{*}{ Mass center $(m)$} & $x$ & -0.1035 & 0.16856 & 0.16856 & 0.096309 & -0.0315 & 0.22344 & 0.22344 \\
\hline & $y$ & 0 & 0 & 0 & 0.001904 & 0 & 0 & 0 \\
\hline & $z$ & 0.034 & 0 & 0 & 0.28128 & -0.02525 & 0 & 0 \\
\hline
\end{tabular}

TABLE III: Parameters of the biped used in the optimal process. $J$ is the inertia tensor measured with respect to reference frame fixed at each body.

$\operatorname{tab} \overline{\overline{\bar{\equiv}}}$

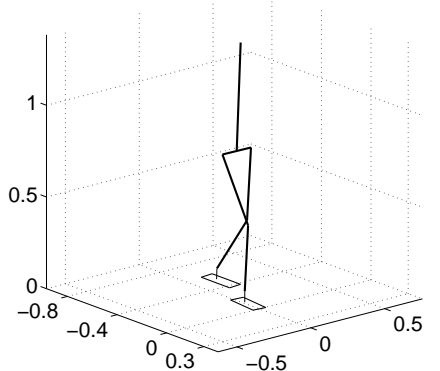

(a) $12.5 \%$ of the half step

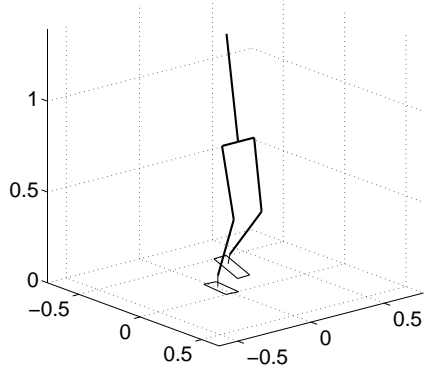

(e) $62.5 \%$ of the half step

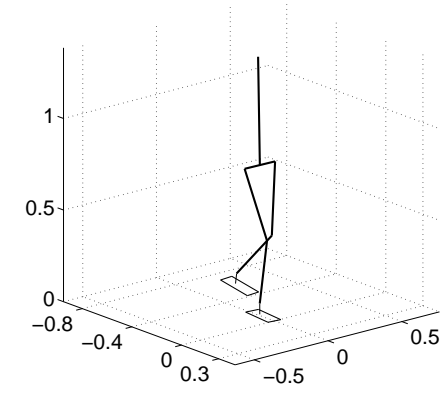

(b) $25 \%$ of the half step

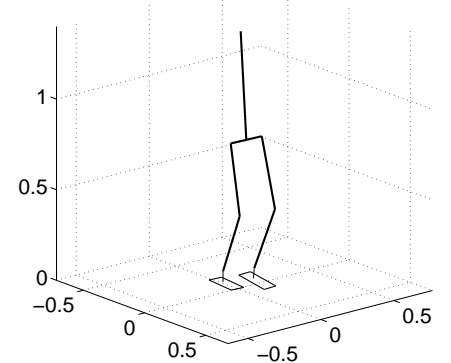

(f) $75 \%$ of the half step

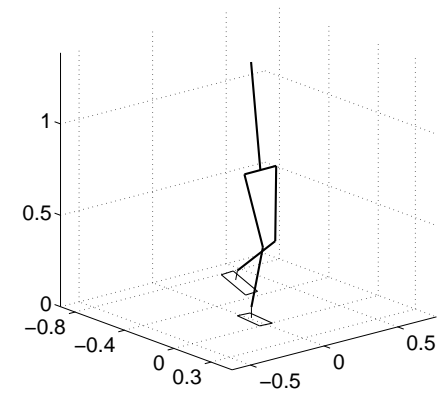

(c) $37.5 \%$ of the half step

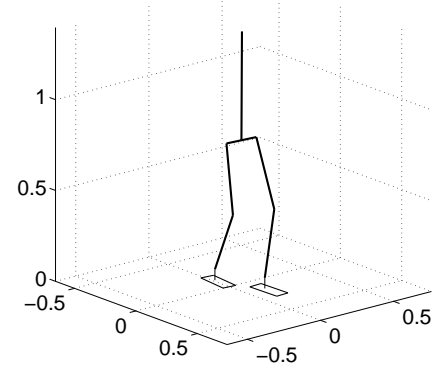

(g) $87.5 \%$ of the half step

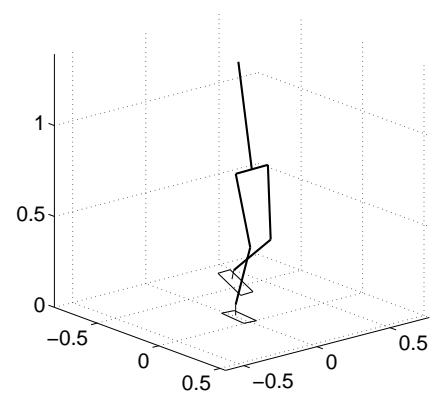

(d) $50 \%$ of the half step

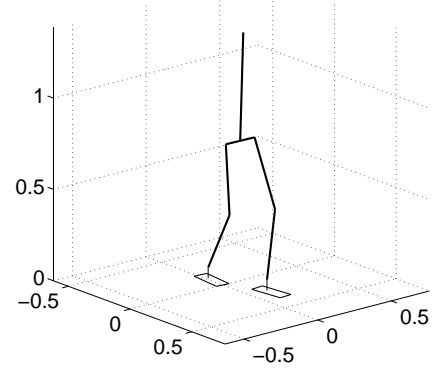

(h) $100 \%$ of the half step

Fig. 4: Stick diagram of the evolution of the biped robot's motion, during one half step, walking at $1.2 \mathrm{~m} / \mathrm{s}$ with a stable gait. figure

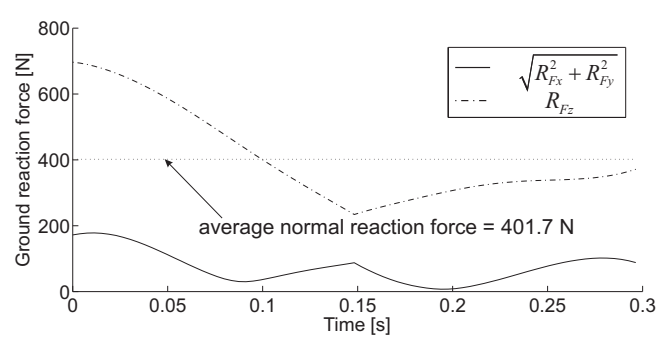

Fig. 5: The ground reaction force during the single support phase. The dash-dotted and solid curve depicts the vertical and the horizontal component of the ground reaction force, respectively. figure

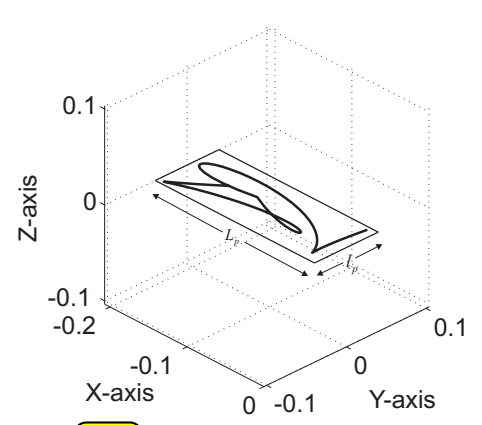

Fig. 6: Location of $\mathrm{C}_{\overline{\overline{\bar{\nu}}}}$ hich evolution satisfies the $\mathrm{Z} \overline{\overline{\overline{2}}}$ riterion. figure 


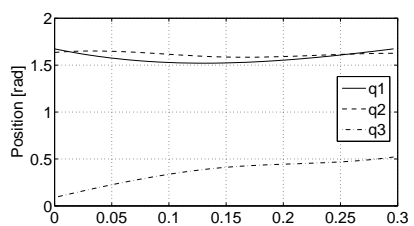

stance ankle joint's positions

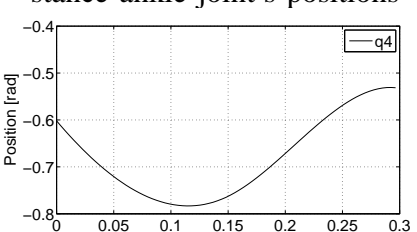

stance knee joint's position

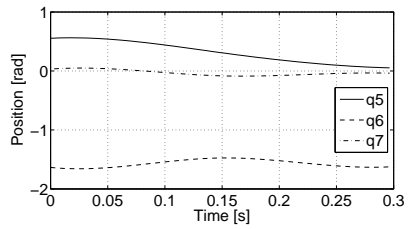

stance hip joint's positions
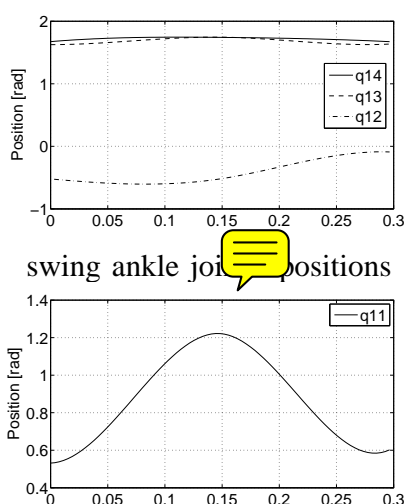

swing knee joint's position

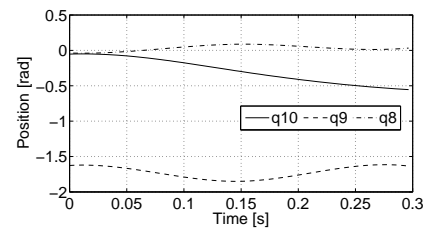

swing hip joint's positions

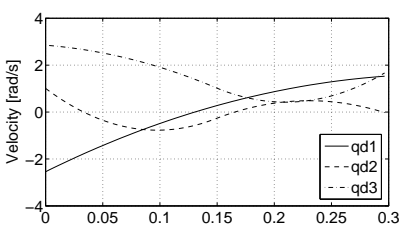

stance ankle joint's velocities

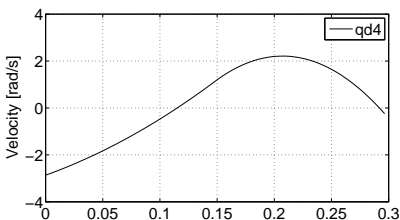

stance knee joint's velocity

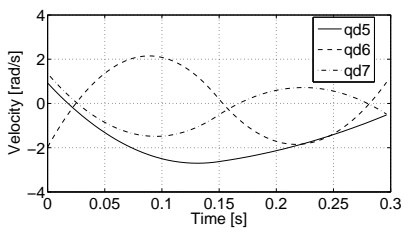

stance hip joint's velocities

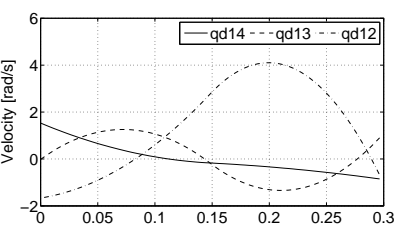

swing ankle joint's velocities
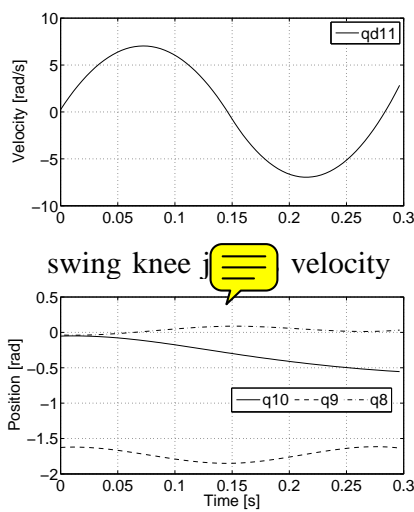

swing hip joint's $\sqrt{\overline{\overline{\bar{E}}}}$ ies

Fig. 7: Evolution of the joint angles and joint velocities versus time. The curves correspond to the stance and swing legs of the robot walking at $1.2 \mathrm{~m} / \mathrm{s}$

figui $\bar{\equiv}$

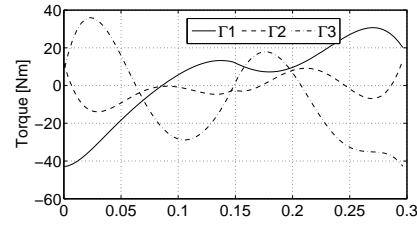

stance ankle joint's torques

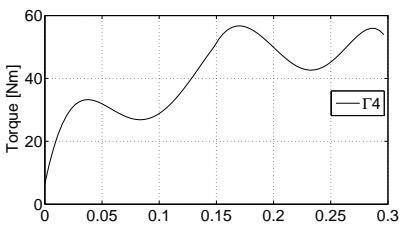

stance knee joint's torque

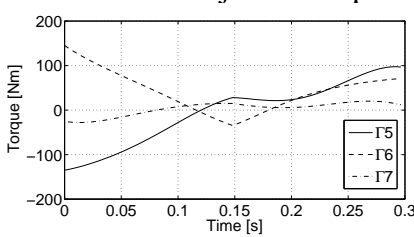

stance hip joint's torques
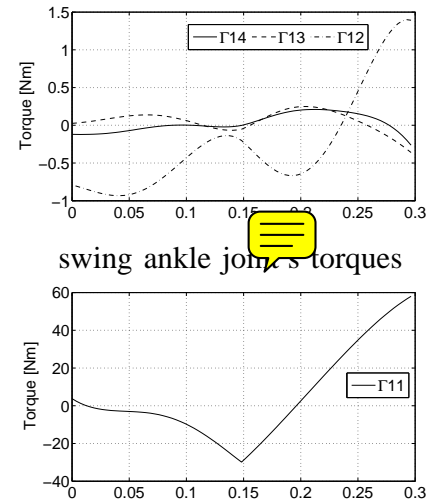

swing knee joint's torque

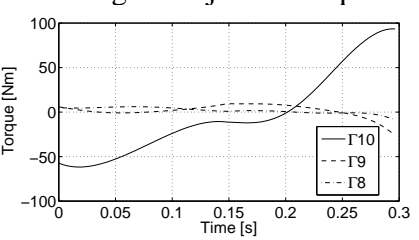

swing hip joint's torques

phases. The Coulomb friction coefficient $\mu$ is $3 / 4$. The average vertical reaction force is $404.37 \mathrm{~N}$, which is coherent with the weight of the biped robot. For this stable gait, the evolution of $\mathrm{C} \equiv$ illustrated in figure 11. This trajectory is the result of the optimization process which evolution remzing within the foot area during the flat-foot sub-phase. The $\overline{\bar{\nu}}_{\text {during }}$ the foot rotation sub-phase is located at the toe, showing a discontinuity during its evolution due to the transition from flat-foot (fully actuated) sub-phase to foot rotation (underactuated) sub-phase. The applied torques are shown in figure 12. Note that the curves have a discontinuity due to the transition from flat-foot sub-phase to foot rotation sub-phase. The torques measured at the stance and swing leg in order to achieve an optimal walk $(1.3 \mathrm{~m} / \mathrm{s})$ with a stable gait describing a rotation about the stance toe, validate the induction of this sub-phase to $y$ ce the energy consumed in the walking. The figure 13 sho he position and velocity states of the robot, walking at $1.3 \mathrm{~m} / \mathrm{s} . q_{0}$ defines the orientation of the biped. During the foot rotation sub-phase the angle, $q_{0}$, increases up to an optimal value $q_{0}=0.435 \mathrm{rd}$.

Fig. 8: The needed joint torques during the motion.

figure

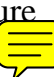

Figure 9 presents the stick-diagram of one step of an optimal walk for the studied three-dimensional bipedal robot. This optimal motion regroups the flat-foot and foot rotation subphases. The introduction of this sub-phase let us obtain an optimal fast motion with a stable gait, which represents the $60.7 \%$ of the total motion. Figure 10 shows the validity of nonsliding and no take-off constraints, during the both sub-

\section{CONCLUSIONS}

In this paper a solution to achieve walking motion with flat-foot and foot rotation sub-phases has been proposed. The studied robot was a three-dimensional biped with geometrical and inertial distribution close to those of the human body. Since the desired motion is based on the solution of an optimal problem and in order to use classical algebraic optimization techniques, the optimal trajectory is defined by a small number of parameters. Some inequality constraints such as the limits 


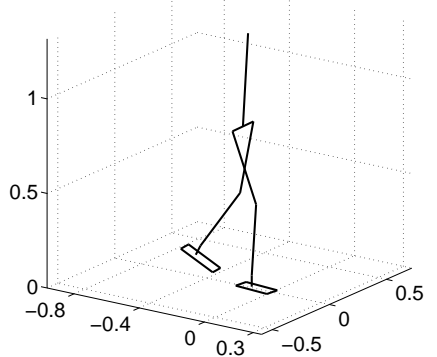

(a) $4.46 \%$ of the half step

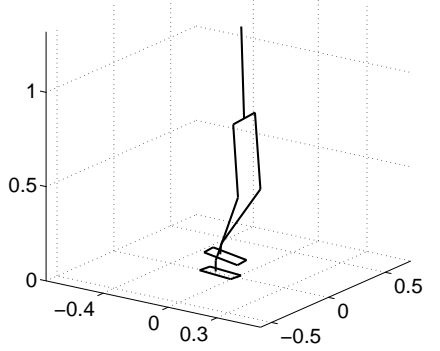

(e) $52.67 \%$ of the half step

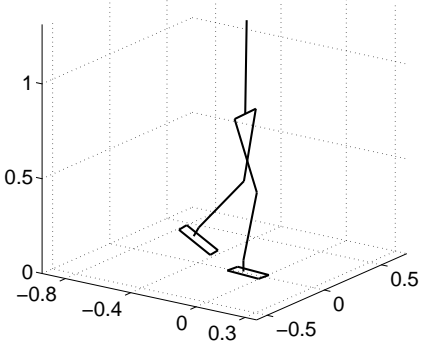

(b) $16.96 \%$ of the half step

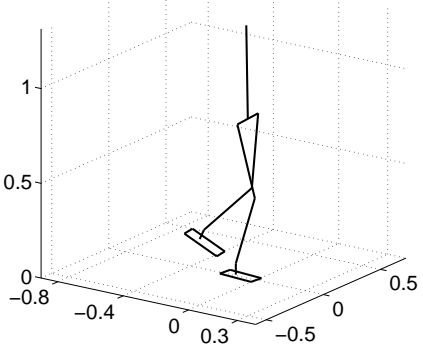

(c) $29.46 \%$ of the half step

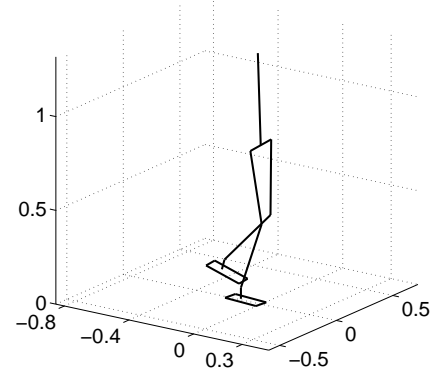

(d) $40.17 \%$ of the half step

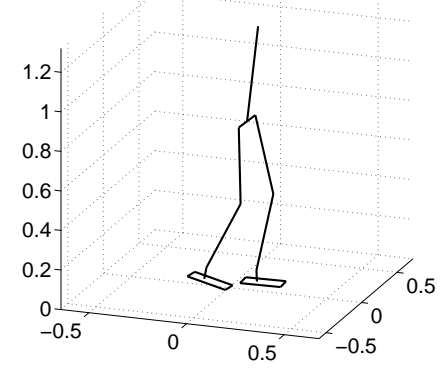

(f) $65.17 \%$ of the half step

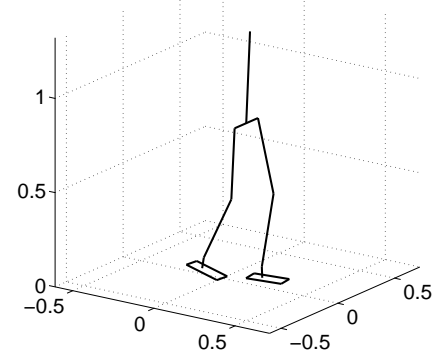

(g) $80.35 \%$ of the half step

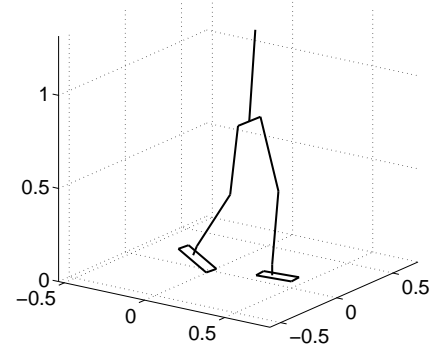

(h) $100 \%$ of the half step

Fig. 9: Stick diagram of the evolution of the biped robot's motion, during one half step, walking at $1.3 \mathrm{~m} / \mathrm{s}$ with a stable gait. In (d) the stance foot begins to rotate about its toe.

figure

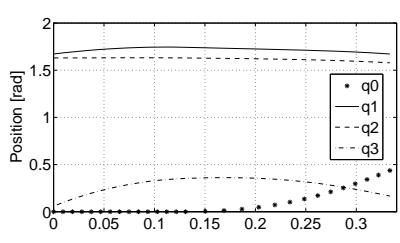

stance ankle joint's positions

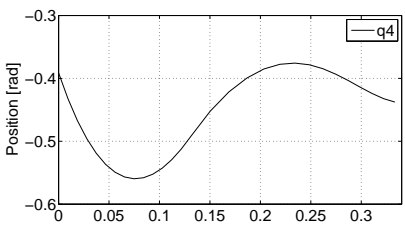

stance knee joint's position

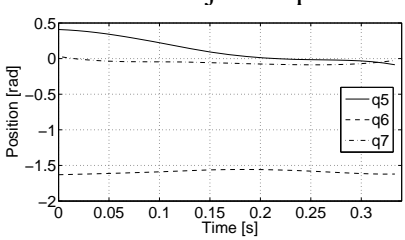

Stance hip joint's positions

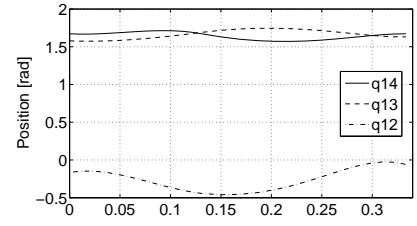

swing ankle joint's positions

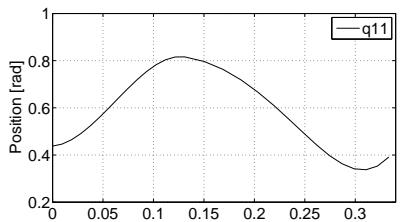

swing knee joint's position

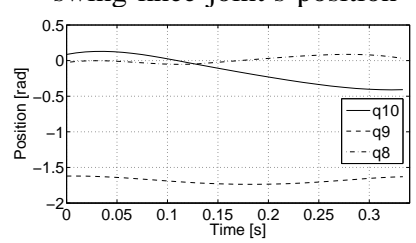

Swing hip joint's positions

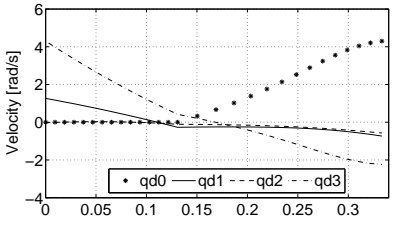

stance ankle joint's velocities

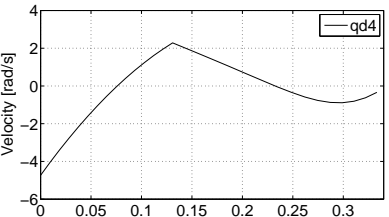

Stance knee joint's velocity

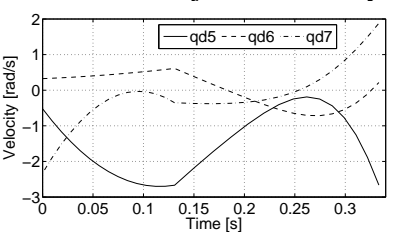

Stance hip joint's velocities

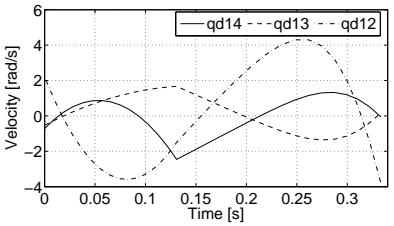

swing ankle joint's velocities

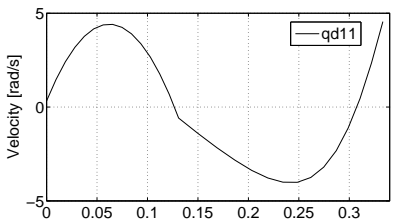

Swing knee joint's velocity

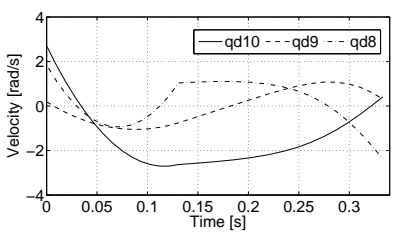

Swing hip joint's velocities

Fig. 13: Evolution of the joint angles and joint velocities versus time. The curves correspond to the stance and swing legs of the robot walking at $1.3 \mathrm{~m} / \mathrm{s}$, where the stance foot rotates with angle, $q_{0}$, about its toe.

figl $\overline{\overline{\bar{\Sigma}}}$

on torque and velocity, the condition of no take-off and no sliding during motion and impact, some limits on the motion of the free leg are taken into account. The desired walking gait was assumed to consist of single supports and instantaneous double supports defined by passive impacts. The single support phase can be composed of a foot rotation sub-phase or not. It is shown that this sub-phase allows to reduce the cost criterion for fast motions. The torques were computed for sampling times using the inverse dynamic model. This model was obtained with the recursive Newton-Euler algorithm. The main contribution of the paper was to extend the optimal trajectories generation of the planar biped robots [10] to an three-dimensional biped robot with rotation of the feet to achieve an optimal fast motion. The developed method has 


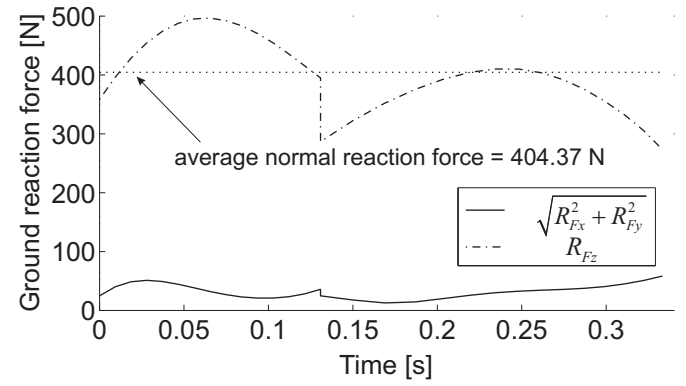

Fig. 10: The ground reaction force during the single support phase. The dash-dotted and solid curve depicts the vertical and the horizontal component of the ground reaction force, respectively. figure

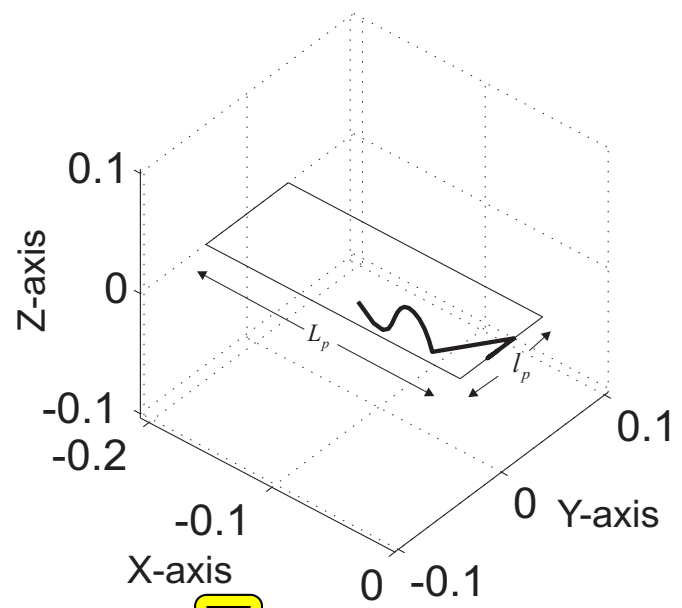

Fig. 11: Location of $\overline{\overline{\bar{\nu}}}$ uring the foot rotation sub-phase, the is located at the stance toe and during the flat-foot sub-phase it is within the stance foot area.

figure

shown that an appropriate choice of the geometric evolution of the robot, corresponding to a motion compatible with the dynamic model, allows to solve the under-actuation sub-phase and to ensure the stability of the robot's motion. Our future study will focus on the introduction of an over-actuated phase to achieve a walking motion where the rear foot rotates around its toe and the front foot rotates around its heel until the foot is flat on the ground.

\section{APPENDIX A}

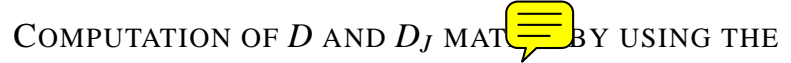 NEWTON-EULER EQUATIONS}

According to the mien de of Walker [29], the inertia matrix, $D$, is calculated by the two recursive calculations of the Newton-Euler algorithm. Using this method we have:

$$
\left[\begin{array}{c}
{ }^{s} \mathbf{f}_{0} \\
{ }^{s} \sqrt{\overline{\bar{\eta}}} \\
\Gamma
\end{array}\right]=D(X)\left[\begin{array}{c}
{ }^{s} \dot{V}_{0} \\
{ }^{s} \dot{\omega}_{0} \\
\ddot{q}
\end{array}\right]+N(X, V)+D_{J} F_{w f} .
$$

In consequence, from this equation, the transpose of the $i^{\text {th }}$ column of $D(X)$ is equal to $\left[{ }^{s} \mathbf{f}_{R},{ }^{s} \mathbf{m}_{R}, \Gamma\right]^{T}$ if

$$
\left[{ }^{s} \dot{V}_{0},{ }^{s} \dot{\omega}_{0}, \ddot{q}\right]^{T}=e_{i}^{T}, V=0, g=0, F_{w f}=0
$$

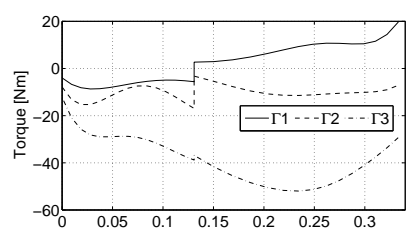

stance ankle joint's torques

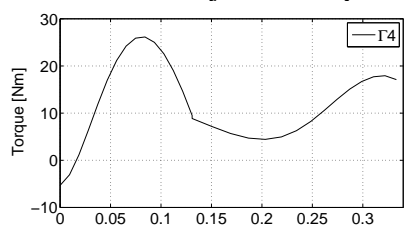

stance knee joint's torque

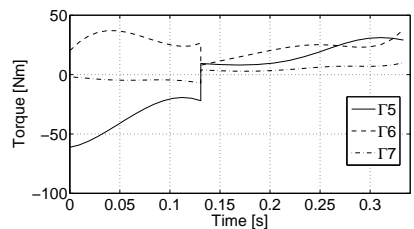

stance hip joint's torques

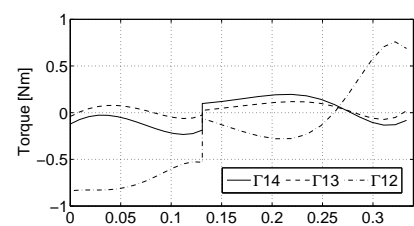

swing ankle joint's torques

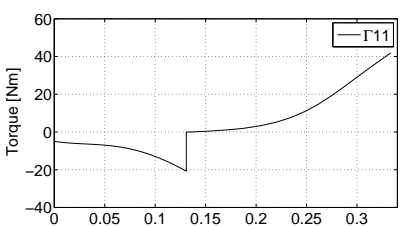

swing knee joint's torque

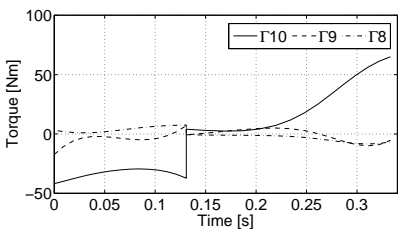

swing hip joint's torques
Fig. 12: The needed joint torques during the motion figure

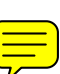

with $e_{i} \in \mathbb{R}^{20}$ the unit vector, whose elements are zero except the $i^{\text {th }}$ element which is equal to 1 .

The Jacobian matrix, $D_{J}$, is calculated by using the same method, by noting from (A-1) that the $i^{\text {th }}$ column is equal to $\left[{ }^{s} \mathbf{f}_{R},{ }^{s} \mathbf{m}_{R}, \Gamma\right]^{T}$ if

$$
\left[{ }^{s} \dot{V}_{0},{ }^{s} \dot{\omega}_{0}, \ddot{q}\right]^{T}=0^{T}, V=0, g=0, F_{w f}=e_{i}
$$

with $e_{i} \in \mathbb{R}^{6}$ the unit vector, whose elements are zero except the $i^{\text {th }}$ element which is equal to 1 .

\section{REFERENCES}

[1] K. Hirai, M. Hirose, T. Takenaka, Y. Haikawa, and T. Takenaka, "The development of honda humanoid robot," Proc. IEEE ICRA, pp. 13211326, 1998

[2] M. Hirose, Y. Haikawa, Y. Haikawa, and T. Takenaka, "Development of humanoid robot asimo," Proc. Inte. Conference on Intelligent Robots and Systems, Workshop2, 2001

[3] M. Gienger, K. Lffler, and F. Pfeiffer, "Towards the design of biped jogging robot," Proc. IEEE ICRA, pp. 4140-4145, 2001.

[4] K. Yokoyama, J. Maeda, T. Isozumi, and K. Kaneko, "Application of humanoid robots for cooperative tasks in the outdoors," Proc. Int. Conference on Intelligent Robots and Systems, Workshop2, 2001.

[5] D. H. Sutherland, K. R. Kaufman, and J. R. Moitoza, Kinematics of normal human gait. Human Walking, Eds. Jessica Rose and James G. Gamble, Williams and Wilkings, 349p, 1994.

[6] K. R. Vaughan, "Rabbit: A testbed for advanced control theory," Crit. Rev. Eng., vol. 12, no. 1, pp. 1-48, 1984.

[7] C. L. Vaugham, B. L. Davis, and J. C. O'Connor, Dynamic of humain gait. Kiboho Publishers, Cape Town, South Africa, 1999.

[8] H. Beaupied, "Etude mécanique et énergétique de la marche, de la course et de lotransition marche $=$ e: influ le la spécialité athlétique" Th $\bar{\equiv}$ doctorat, Univers $\overline{\overline{\bar{\equiv}}}$ Jantes/d $\overline{\overline{\bar{E}}}$ entrale de Nantes, Renne II, Prance, October 2003.

[9] J. H. Choi and J. Grizzle, "Planar bipedal walking with foot rotation," In Proc. of the 2005 American Control Conference, Portland, OR, pp. 4909-4916, 2005.

[10] D. Tlalolini, C. Chevallereau, and Y. Aoustin, "Optimal reference motions with rotation of the feet for a biped," Proc. IDETC/MR, 2008. 
[11] C. Chevallereau, G. Abba, Y. Aoustin, F. Plestan, E. R. Westervelt, C. Canudas-de Witt, and J. W. Grizzle, "Rabbit: A testbed for advanced control theory," IEEE Control System Magazine, vol. 23, no. 5, pp. 5778, 2003.

[12] C. Chevallereau, F. A. M., and D. Djoudi, "Tracking of a joint path for the walking of an under actuated biped," Robotica, vol. 22, no. 1, pp. $15-28,2004$.

[13] W. Khalil and J. Kleinfinger, "A new geometric notation for open and closed loop robots," Proc. of the IEEE Conference on Robotics and Automation, pp. 1174-1180, 1985.

[14] W. Khalil and E. Dombre, Modeling, identification and control of robots. Hermes Sciences Europe, 2002.

[15] M. J.Y.S.Luh and R.C.P.Paul, "Resolved-acceleration control of mechanical manipulators," IEEE Trans. on Automatic Control, vol. 25, no. 3, pp. 468-474, 1980.

[16] M. Vukobratovic and B. Borovac, "Zero-moment pont-thirty five years of its life," International Journal of Humanoid Robotics, vol. 1, no. 1, pp. 157-173, 2004.

[17] M. Vukobratovic and Y. Stepanenko, "On the stability of anthropomorphic systems," Mathematical Biosciences, vol. 15, pp. 1-37, 1972.

[18] M. Vukobratovic, B. Borovac, D. Surla, and D. Stokic, Biped Locomotion. Springer-Verlag, 349p, 1990.

[19] A. Formal'sky, Locomotion of Anthropomorphic Mechanisms. Nauka, Moscow [In Russian], 1982.

[20] E. Westervelt, J. Grizzle, C. Chevallereau, J. Choi, and B. Morris, Feedback Control of Dynamic Bipedal Robot Locomotion. Taylor \& Francis/CRC Press, 2007.

[21] C. Chevallereau and Y. Aoustin, "Optimal reference trajectories for walking and running of a biped," Robotica, vol. 19, no. 5, pp. 557$569,2001$. 